\title{
The Dredging Layout Scheme Research of Xigang Channel in Dongliu Waterway
}

\author{
Liang $\mathrm{SHU}^{1, \mathrm{a}}$ \\ ${ }^{1}$ Changjiang Nanjing Waterway Engineering Bureau, Nanjing 210011, China \\ ashuliang01@126.com
}

Keywords: Dongliu Waterway, Mathematical Model, Xigang Waterway, Dredging Scheme.

\begin{abstract}
In order to determine reasonable channel layout and maintenance the dredging areas, this paper set up mathematics model of two-dimensional water flow for channel, and the numerical simulation results are verified by the observed data. Based on this, the fluid field change laws of Xigang channel in flood, median and low water levels are analyzed, the dredging layout scheme of Xigang channel is proposed, and the water velocity changes in Xigang channel before and after dredging maintenance are compared. The results show that the following dredging layout scheme is reasonable, the entrance of Xigang channel is located at main flow area of Dongliu waterway, the left border line of Xigang channel is close to the channel regulation project and the main channel from Tiansha islet to the downstream, the right boundary will depend on the wide requirements of the channel, and the channel outlet can smoothly connect to water flow.
\end{abstract}

\section{Introduction}

Dongliu waterway is one of the important navigation-obstructing shoals in the lower reaches of the Yangtze River. The translocation of the main and branch of the waterway occurs frequently, and the riverbed evolution is complicated. The waterway regulation project which has been implemented is difficult to fundamentally solve the problems existed in the waterway channel. In August 2013, due to a large amount of sediment from the upper reaches of the Yangtze River and the rapid decline of the water level of the Yangtze River and the shoals depression, the water level of Dongliu waterway declined nearly $1.5 \mathrm{~m}$ within half a month and presents a continuous downward trend and the navigation conditions of West Port channel deteriorated, causing a certain threat to the navigational safety of the ships. The Yangtze river Waterway Engineering Bureau in Nanjing sent "HangJun16" wheel to the field maintenance, and timely adjust "HangJun8" to dredge, and then sent "Long Lion 6" cutter suction dredger to participate in the maintenance of Dongliu waterway, which effectively alleviate the pressure of Dongliu waterway maintenance. However, according to the November 26, 2013 mapping, "Long Lion 6" dredging channel from 6\# red floating following sustained the sediment from upstream and Tiansha Sandbar extrusion becomes shallow. Dredging and red floating connection slot near the shallow area after initial digging deep becomes shallow gradually, and the excavated depth within the initial $4 \mathrm{~m}$ continuous deposition becomes shallow, silting thickness up to $2 \sim 3 \mathrm{~m}$. With the arrival of the flood period in 2014, the maintenance situation of the Dongliu waterway is still very serious. In order to grasp the cause of the deterioration of the Xigang channel, study the reasonable maintenance dredging scheme, ensure the smooth flow of Dongliu waterway, this paper established 2-D flow mathematical model to optimize and analyse the Xigang channel layout from the angle of flow field.

\section{Establishment of Mathematical Model}

\section{Basic Equations}

Continuity equation of water flow:

$$
\frac{\partial H}{\partial t}+\frac{1}{C_{\xi} C_{\eta}} \frac{\partial}{\partial \xi}\left(h u C_{\eta}\right)+\frac{1}{C_{\xi} C_{\eta}} \frac{\partial}{\partial \eta}\left(h v C_{\xi}\right)=0
$$


Momentum equation in $\xi$ direction:

$$
\begin{aligned}
& \frac{\partial u}{\partial t}+\frac{1}{C_{\xi} C_{\eta}}\left[\frac{\partial}{\partial \xi}\left(C_{\eta} u^{2}\right)+\frac{\partial}{\partial \eta}\left(C_{\xi} v u\right)+v u \frac{\partial C_{\eta}}{\partial \eta}-v^{2} \frac{\partial C_{\eta}}{\partial \xi}\right]=-g \frac{1}{C_{\xi}} \frac{\partial H}{\partial \xi}+f v \\
& -\frac{u \sqrt{u^{2}+v^{2}} n^{2} g}{h^{4 / 3}}+\frac{1}{C_{\xi} C_{\eta}}\left[\frac{\partial}{\partial \xi}\left(C_{\eta} \sigma_{\xi \xi}\right)+\frac{\partial}{\partial \eta}\left(C_{\xi} \sigma_{\eta \xi}\right)+\sigma_{\xi \eta} \frac{\partial C_{\xi}}{\partial \eta}-\sigma_{\eta \eta} \frac{\partial C_{\eta}}{\partial \xi}\right] .
\end{aligned}
$$

Momentum equation in $\eta$ direction:

$$
\begin{aligned}
& \frac{\partial v}{\partial t}+\frac{1}{C_{\xi} C_{\eta}}\left[\frac{\partial}{\partial \xi}\left(C_{\eta} v u\right)+\frac{\partial}{\partial \eta}\left(C_{\xi} v^{2}\right)+u v \frac{\partial C_{\eta}}{\partial \xi}-u^{2} \frac{\partial C_{\xi}}{\partial \eta}\right]=-g \frac{1}{C_{\eta}} \frac{\partial H}{\partial \eta}-f u \\
& -\frac{v \sqrt{u^{2}+v^{2}} n^{2} g}{h^{4 / 3}}+\frac{1}{C_{\xi} C_{\eta}}\left[\frac{\partial}{\partial \xi}\left(C_{\eta} \sigma_{\xi \eta}\right)+\frac{\partial}{\partial \eta}\left(C_{\xi} \sigma_{\eta \eta}\right)+\sigma_{\eta \xi} \frac{\partial C_{\eta}}{\partial \xi}-\sigma_{\xi \xi} \frac{\partial C_{\xi}}{\partial \eta}\right] .
\end{aligned}
$$

In the formular, $\xi, \eta$ respectively represents the two orthogonal curvilinear coordinate in orthogonal curvilinear coordinates; $\mathrm{u}, \mathrm{v}$ respectively represents the velocity along $\xi, \eta$ direction; $\mathrm{h}$ reprents the depth; $\mathrm{H}$ represents the water level; $\mathrm{f}$ reprents the Coriolis Coefficient; $C_{\xi}, C_{\eta}$ represent the Lame Coefficient in orthogonal curvilinear coordinates:

$$
\begin{gathered}
C_{\xi}=\sqrt{x_{\xi}^{2}+y_{\xi}^{2}}, C_{\eta}=\sqrt{x_{\eta}^{2}+y_{\eta}^{2}} . \\
\sigma_{\xi \xi}, \sigma_{\xi \eta}, \sigma_{\eta \xi}, \sigma_{\eta \eta} \text { represent the Reynolds Stress: } \\
\sigma_{\xi \xi}=2 v_{t}\left[\frac{1}{C_{\xi}} \frac{\partial u}{\partial \xi}+\frac{v}{C_{\xi} C_{\eta}} \frac{\partial C_{\xi}}{\partial \eta}\right] \quad \sigma_{\eta \eta}=2 v_{t}\left[\frac{1}{C_{\eta}} \frac{\partial v}{\partial \eta}+\frac{u}{C_{\xi} C_{\eta}} \frac{\partial C_{\eta}}{\partial \xi}\right] \\
\sigma_{\xi \eta}=\sigma_{\eta \xi}=v_{t}\left[\frac{C_{\eta}}{C_{\xi}} \frac{\partial}{\partial \xi}\left(\frac{v}{C_{\eta}}\right)+\frac{C_{\xi}}{C_{\eta}} \frac{\partial}{\partial \eta}\left(\frac{u}{C_{\xi}}\right)\right]
\end{gathered}
$$

$v_{t}$ represents the Turbulent Viscosity Coefficient.

\section{Numerical Solution}

The equation (1) (3) expressed by the following general format:

$$
C_{\xi} C_{\eta} \frac{\partial \psi}{\partial t}+\frac{\partial\left(C_{\eta} u \psi\right)}{\partial \xi}+\frac{\partial\left(C_{\xi} v \psi\right)}{\partial \eta}=\frac{\partial}{\partial \xi}\left(\Gamma \frac{C_{\eta}}{C_{\xi}} \frac{\partial \psi}{\partial \xi}\right)+\frac{\partial}{\partial \eta}\left(\Gamma \frac{C_{\xi}}{C_{\eta}} \frac{\partial \psi}{\partial \eta}\right)+C \text {. }
$$

Only need to compile (4) into a general program, all control equations are available to be solved in this program. $\Gamma$ is the Diffusion coefficient; $C$ is the source term. According to the characteristics of the control equations, the longitudinal velocity $\mathrm{u}$, lateral velocity $\mathrm{v}$, water depth $\mathrm{h}$ and other physical quantities are not arranged in the same grid in order to decorate staggered grid, and make the inlet and outlet boundary through the calculation of longitudinal velocity points , solid wall through the calculation of lateral velocity points, and the density of the grid depends on the changes in the physical quantities, and discrete control equations by using control volume method.

\section{Definite Conditions}

The initial water level field can be carried out using linear interpolation with calculation domain, downstream water level and section spacing, and it cannot consider the transversal slope in the cross 
section. As the computational domain is longer, give the water level of a few sections in two-dimensional domain using the measures to calculate the water surface line, then do linear interpolation piecewise.

For the initial velocity field, make $\mathrm{v}=0$ to $\eta$ direction, $\mathrm{u}$ calculated by manning formula to $\xi$ direction, and then do correction of the total flow rate in section. Other boundary conditions processing includes: land boundary water level are given according to boundary conditions $\left(\frac{\partial}{\partial n}=0\right)$ of normal direction, shore boundary velocity as zero.

\section{Numerical Model Examination}

\section{Validation of the Calculation Conditions}

The initial calculation is based on the measured terrain that was observed by the Yangtze River Waterway Bureau in 2010 April, and calculated flow uses the bankfull discharge, the upstream flow rate of $38895 \mathrm{~m}^{3} / \mathrm{s}$, the downstream water level of $10.99 \mathrm{~m}$, the position of the measurement point as shown in figure 1 .

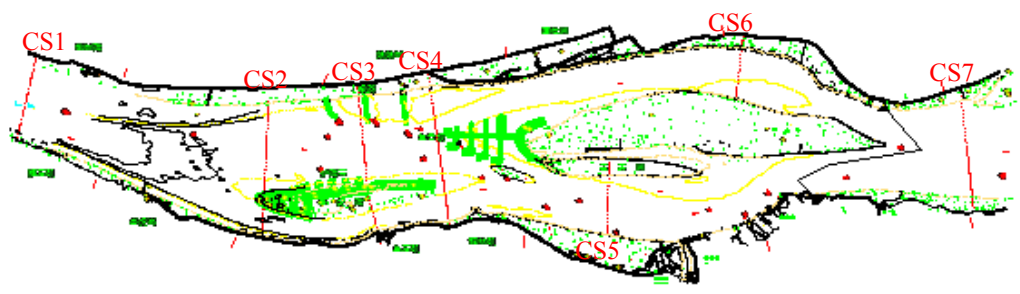

Fig.1 Water Velocity Verification Point

A two-dimensional mathematical model computing grid is based on the river-regime-fitted orthogonal grid. The grid number is $250 \times 80$. The grid spacing is $100 \mathrm{~m} \sim 130 \mathrm{~m}$ in the flow direction, and the grid spacing is $10 \mathrm{~m} \sim 30 \mathrm{~m}$ in vertical flow direction. Calculate in the reach of roughness by hydrological data in reverse, and debug in unit block according to the local terrain. To reflect the changes of the different flows and boundary locations using "cutting", "cutting" the bed elevation of the exposed unit dropped to below the surface, and obligate thin layer depth, at the same time, change the unit roughness ( $\mathrm{n}$ take 10 orders of magnitude), make the exposed unit $\mathrm{u}, \mathrm{v}$ calculated value automatically for 0 , in order to ensure the continuation and normal of mathematical model calculation.

\section{Validation of the Water Surface Line}

Figure 2 is respectively for the water level change on the left side of the Dongliu waterway. It can be seen that calculated values are relatively closed to the measured values, the largest difference within $10 \mathrm{~cm}$, and the resistance of the model can reflect the change of the prototype.

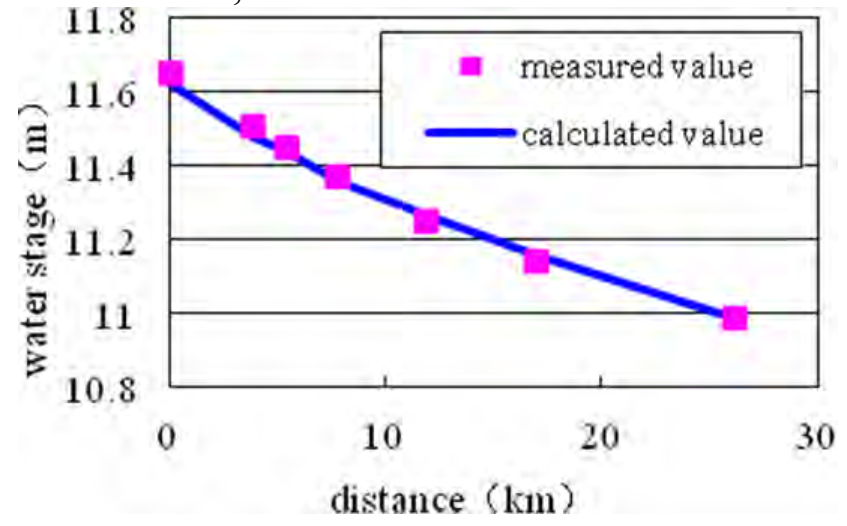

Fig. 2 Water Level Change of the Left Side

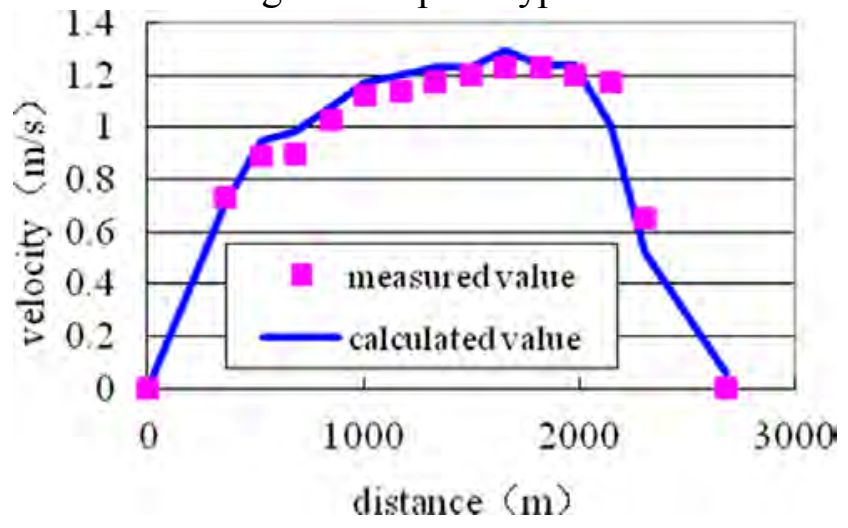

Fig.3 The CS3 Section Velocity Distribution 


\section{Validation of the Section Velocity Distribution}

Figure 3 is the test results of the average vertical velocity distribution in the typical section. By the graph, you can see that calculation velocity distribution is basically identical with actual situation.

\section{Flow Pattern Analysis of Engineering Area}

\section{Engineering Area Flow Field under the Typical Flow of Water}

Flow is in one of the important indices for waterway and chamfered direction arrangement. This study is based on the latest terrain data which respectively to carry out the flow field calculation under the situation of flood, moderate flow and low flow. According to the results of flow pattern, it should choose the area which has a small intersection angle with the flow direction as the direction of the channel and the chamfer under the condition of keep the depth of the water. The hydrological conditions of the flow field calculation as shown in table 1 .

Tab. 1 The Hydrological Conditions of the Flow Field Calculation

\begin{tabular}{|c|c|c|c|c|}
\hline $\begin{array}{c}\text { Working } \\
\text { condition }\end{array}$ & Flow $\left(\mathrm{m}^{3} / \mathrm{s}\right)$ & $\begin{array}{c}\text { The downstream } \\
\text { water level }(\mathrm{m})\end{array}$ & $\begin{array}{c}\text { Time of } \\
\text { occurrence }\end{array}$ & Note \\
\hline 1 & 58300 & 14.941 & 2010.8 .6 & Flood \\
\hline 2 & 38895 & 10.99 & 2010.4 .22 & Moderate flow \\
\hline 3 & 13200 & 5.375 & 2010.11 .23 & Low flow \\
\hline
\end{tabular}

The engineering area flow pattern with a typical condition is shown Figure 4. In the flood condition, the flow from upstream is affected by Laohutan beach and Lianhua sandbar. Mainstream is divided into three shares, a share is located in Donggang waterway, a port is located in the Xigang waterway, and another is located in the Lanhua waterway. The mainstream located in Xigang waterway is affected by the left boundary of Lianhua sandbar which flows towards the Tianyu ditch and it has an intersection angle with a river between Tiansha sandbar and Laohutan sandbar. In the moderate flow condition, the flow of Xigang waterway turns to the right, the direction of the river between Tiansha sandbar and Laohutan sandbar is same as the direction of flow. In the low flow condition, because the downstream water level is low, flow coming into main channel, especially the Xigang waterway, the elevation of Laohutan's tail is high, so water cannot flow through it and a large proportion of water flow into downstream through Tianyu ditch. According to the analysis, in different condition, the mainstream of Xigang waterway always flow through Tianyu ditch, so mud is deposited easily at the channel between the tail of Laohutan sandbar and Tiansha sandbar.

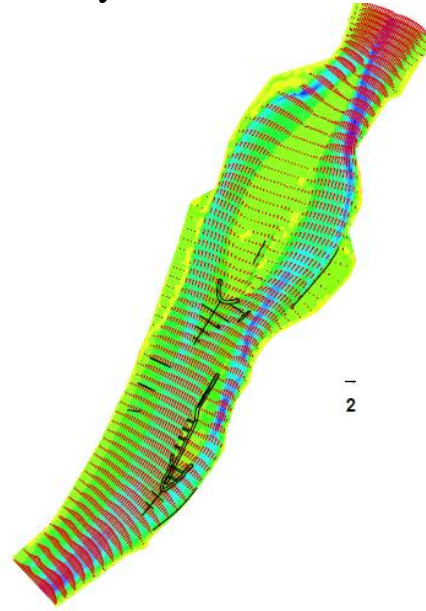

(a)Flood

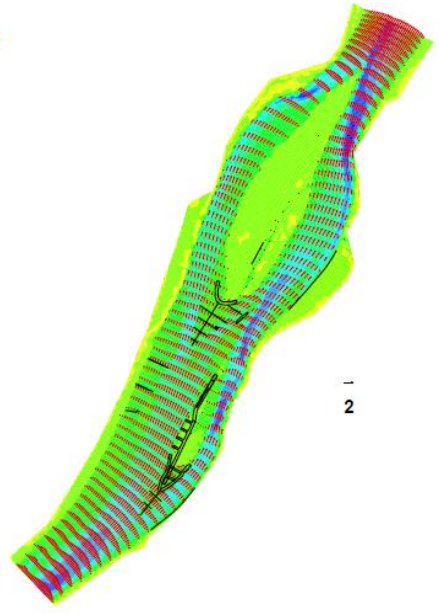

(b)Moderate Flow

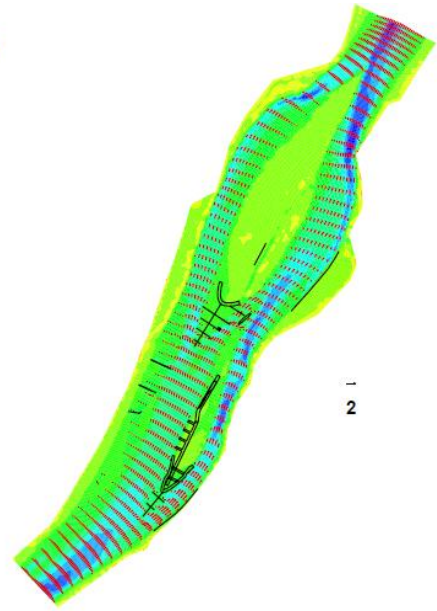

(c)Low Flow

Fig.4 The Engineering Area Flow Pattern under a Typical Condition 


\section{Waterway and Dredging Channel Layout Scheme and the Distribution of Velocity}

According to the results of flow field calculations above, under the condition of different quantity of flow, the flow of Xigang waterway tends to Tianyu ditch, because of the flow condition of this area is better. Based on the measured topographic map in 2014, Xigang waterway left line may be arranged close to Tiansha sandbar and regulation structure, the right boundary will depend on the wide requirements of the channel, narrowest width of about $120 \mathrm{~m}$, the channel is basically consistent with the trend towards the water. Channel layout scheme is shown in Figure 5.

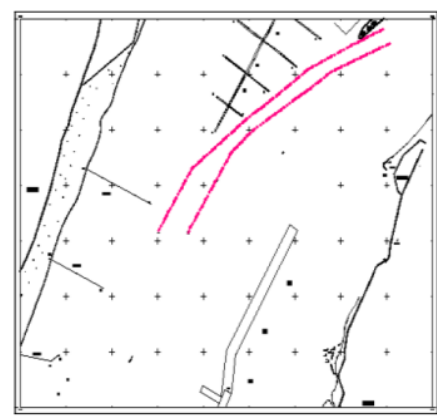

Fig. 5 Channel

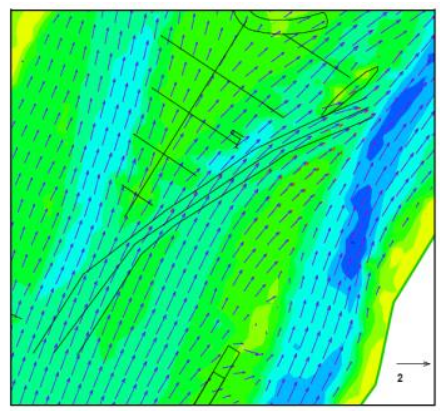

Fig. 7 Comparisons of Flow Velocity between the Channel before and after Dredg When Moderate Flow

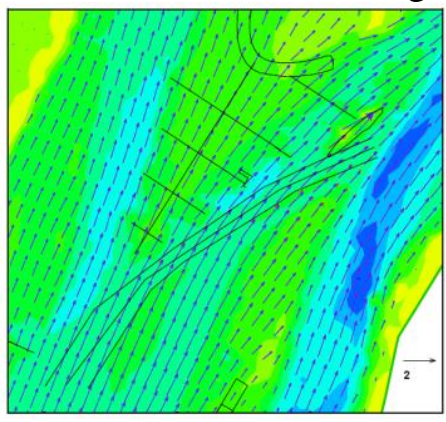

Fig. 6 Comparisons of Flow Velocity between the Channel before and after Dredging When Flood

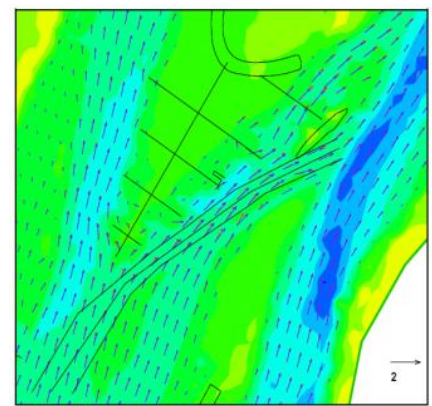

Fig. 8 Comparisons of Flow Velocity between the Channel before and after Dredging When Low Flow

The calculation of the inlet and outlet boundary condition control as shown in Table 1 which respectively analyzed under the situation of flood, moderate flow and low flow. Xigang waterway flow field under the no dredging condition and under the maintenance design water depth $4.5 \mathrm{~m}$ after dredging were calculated based on the terrain conditions for the newest terrain of 2014 July and the design scheme of dredging respectively.

The comparison of velocity between before the construction and after the construction of the Xigang waterway area is respectively analyzed under the condition of flood, moderate flow and low flow which is shown form figure 6 to 8 . It can be seen that, the flow direction of channel has a small intersection angle between channel and dredged channel. At flood period and median water period, the variation of flow velocity of channel and dredged channel before and after dredging is little. The flow velocity is slightly decreased after dredging and the flow direction tends to turn right. At low flow period, the mainstream comes into channel and the flow velocity increases so it is benefit for channel to scour.

\section{Conclusions}

This paper establishes a 2-D mathematical model of Dongliu waterway, the measured data verification results show that the model can reflects the motion law of flow. The changes of the flow field in different conditions were calculated by using the model of Xigang waterway: in the condition of flood, the mainstream of Xigang waterway is affected by the left of Laohutan sandbar, 
the flow tends to straight, directly flow into Tianyu ditch, the channel between Tiansha sandbar and the tail of Laohutan sandbar has an angle with mainstream. In the condition of moderate flow, the flow of Xigang waterway turns to right and the direction of the channel between Tiansha sandbar and Laohutan sandbar is same as the direction of flow. In the condition of low flow, flow comes into main channel and the mainstream of Xigang waterway always flow through Tianyu ditch. So that the Xigang waterway left line should be close to the Tiansha sandbar and regulating structure, the right boundary is considered the necessary width and narrowest width of about $120 \mathrm{~m}$, the channel is basically consistent with the trend towards the water.

\section{References}

[1]Wenquan LI, Xinmin TU, Zuxin YANG, Hongchun LIU: Analysis of river bed evolution and discussion on waterway regulation thought of Dongliu channel on the lower reach of the Yangtze River, Port and Waterway Engineering (2011.10).

[2]Hongchun LIU, Wei ZHANG, Wenquan LI, Peng WAN: Evolution characteristics of the left beach of Dongliu waterway and its effect on waterway condition, Port and Waterway Engineering (2013.08).

[3]Hong CHEN, Shengan WANG: Two dimensional flow mathematical simulation and its application in complicated channel,Jounal of Fuzhou University(Natural Science),(2010.08)

[4]Youxiang CAI, Cen WEN: Application of 2D Flow Mathematical Model in Flood Control Evaluation of Tongjiang River Bridge, Journal of Water Resources and Architectural Engineering (2012.12). 